\title{
Right Vertebral Artery Spontaneous AVF Mimicking as Spinal Extradural Tumour: A Case Report
}

\author{
Taher $\mathrm{T}^{1}$, Haroon $\mathrm{K}^{2}$, Alam $\mathrm{S}^{3}$, Alamgir $\mathrm{A}^{3}$, Raihan $\mathrm{F}^{4}$, Reza $\mathrm{MA}^{5}$, Hossain $\mathrm{SS}^{6}$
}

Conflict of interest: There is no conflict of interest relevant to this paper to disclose.

Funding Agency: Was not funded by any institute or any group.

Contribution of Authors: Taher T was Principal investigator, Haroon K help for protocol preparation, Alam S, Alamgir A, Raihan F, Reza MA help for data collection and Hossain SS help for editorial formatting.

Copyright: @2019 Bang. JNS published by BSNS. This article is published under the creative commons CC-BY-NC license.

This license permits use distribution (https://creativecommons.orgf/ licences/by-nc/4-0/) reproduction in any medium, provided the original work is properly cited, and is not used for commercial purposes.

Received: 07 January, 2019

Accepted: 21 January, 2019

\begin{abstract}
:
We report a case of a 20 years old female patient, who presented with the complaints of progressive quadriparesis which eventually led to quadriplegia. Her weakness started from the right side of the body and then to the left. Her MRI of the neck revealed an extradural lesion extending from the $C 2$ up to C6 vertebral level. It had compressed the dural tube to the left. The lesion continued to the neck through the inervertebral foramen at C6. Her CT angiogram of the neck vessels showed an AVM arising from the right SCA and entering the Spinal canal at C6 level and draining into the right IJV at $C 2$ level. She underwent surgery and the AVF was ligated at the origin. The AVM had collapsed. After 15 days following surgery, she had significant improvement of symptoms. After one and half months she could walk with support.
\end{abstract}

Key Words: AVM, Vertebral artery, cystic schwannoma, quadriparesis, Subclavian artery, AVF

\section{Abbreviations:}

SCA-Subclavian artery, VA- Vertebral Artery, IJV-Internal Jugular vein, SVM- Spinal vascular malformation, AVF-Arteriovenous Fistula, AVM- Arteriovenous malformation

Bang. J Neurosurgery 2019; 9(1): 69-74

\section{Introduction:}

Vertebral arteriovenous fistula (AVF) is a rare vascular disease characterised by abnormal connections between the extracranial vertebral artery (VA) or its branches and the neighboring veins. Many vertebral AVFs are of traumatic origin, following incidents like penetrating neck injuries or medical interventions such as carotid artery or VA puncture. Non-traumatic vertebral AVFs are usually divided into congenital and spontaneous types. The pathogenetic mechanism of spontaneous fistulas is usually unknown, but there may be a relationship with neurofibromatosis ${ }^{1}$.

Spinal vascular malformations (SVM) are rare and still under diagnosed entities that typically lead to progressive spinal cord symptoms and myelopathy if not properly treated ${ }^{2}$. The space-occupying nature of some of these lesions and a circulatory"Steal" phenomenon are additional possible pathomechanisms ${ }^{2}$.

Amirjamshidi had reported about traumatic AVF in the neck and cerebral vessels. According to him arterial injuries can lead to acute hemorrhage, complete major vessel obstruction, and traumatic aneurysm (TA) or arteriovenous fistula (AVF) formation ${ }^{3}$. Ross had also reported a post traumatic vertebral artery pseudoaneurysm at C4-C5 level ${ }^{4}$.

Geering in 1976 had commented that, because of its rarity arteriovenous fistula of the vertebral artery may be missed easily, even in the presence of a cardiac murmur. The only way to suspect such a lesion is by auscultation and palpation of the head and neck area, and this is not everyone's routine. A correct diagnosis

1. Dr. Tania Taher, Assistant Professor, HFRC Medical College Hospital

2. Dr. Kaisar Haroon, Assistant Professor, Department of Neurosurgery, NINSH.

3. Dr. Md. Shafiul Alam, Dr. Abdullah Alamgir, Associate Professor, Department of Gamma Knife, NINSH.

4. Dr. Farid Raihan, Resident, Department of Neurosurgery, NINSH

5. Dr. Arif Reza, Medical Officer, Department of Neurosurgery, NINSH

5. Prof. Sk. Sader Hossain, Professor and Head, Department of Neurosurgery, NINSH

Address of Correspondence: Dr. Kaisar Haroon, Assistant Professor, Department of Neurosurgery, NINS. Email: kaisar298@gmail.com, Phone: +8801711196577. ORCiD: 0000-0002-3065-7877 
however is imperative for proper therapy, which consists of surgical removal of the fistula to prevent cardiac failure at a later stage ${ }^{5}$.

Arteriovenous malformations represent the persistence of embryonic arteriovenous shunts. During the first period of embryological development of the blood vessels of the central nervous system, primordial endothelial blood-containing channels develop. These are neither arteries nor veins, but rather a germinal bed of endothelium. At a later stage, arteries and veins form through fusion and dissolution of a later-developed capillary mesh. An immature vascular circuit becomes functional in the 3- to 5-week embryo. The stems of originof all cerebral arteries in the 2-month old embryoare similar to the adult. The veins reach maturity in the 3-month old embryo. However, changes in the venous system of the brain continue until birth. It can be seen why a minor aberration occurring in early development may lead to a gross anomaly of the vascular system. The vertebral artery evolves as a longitudinal arterial channel from segments of a series of transversely running arteries and the anastomosis between them. At its origin, there is a segment of the seventh cervical segmental artery, distal to the origin of the primitive subclavian artery. The vertebral artery is formed by the $32^{\text {nd }} d a y^{6}$. May be that is why there is spontaneous AVF of the vertebral artery.

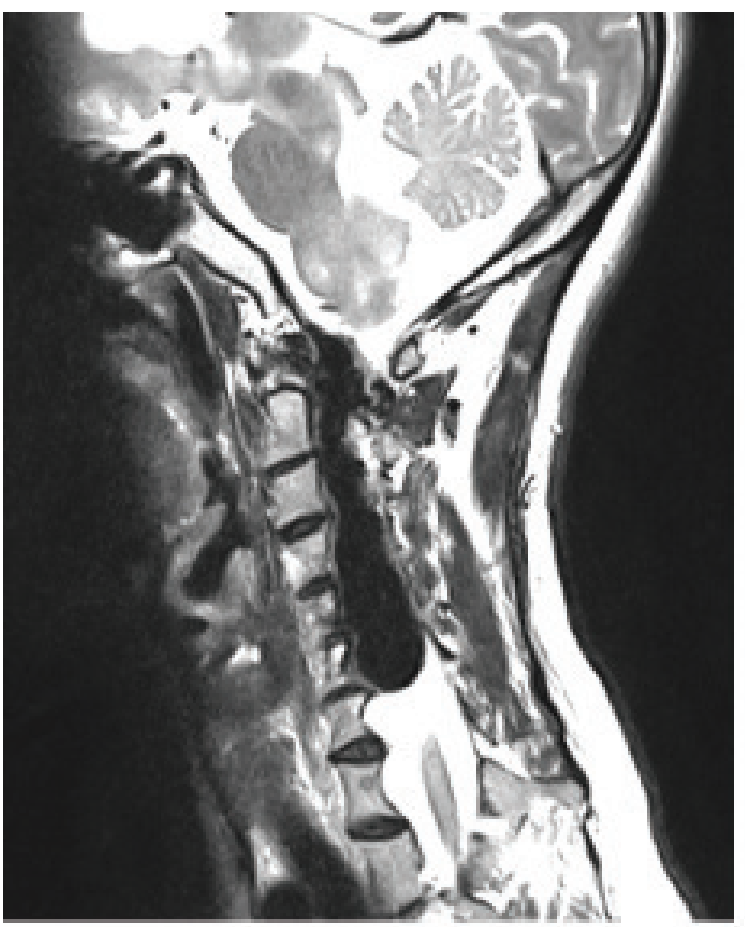

\section{Case Report:}

A twenty years old female, presented to our department with gradual weakness of all four limbs for two months. It started on the right side and then involved the left side. Now all her limbs were weak. Her bladder is now involved. She had no history of trauma or fever. On examination, higher psychic function and speech was normal, but she was unable to walk. She had no sensory level. Muscle power was $3 / 5$ in left side and $2 / 5$ on the right side. All her cranial nerves were intact. There were no signs of cerebeller dysfunction or meningeal irritation. There was a palpable thrill on the right supraclavicular region and bruit was also heard over the right SCA. She had no Horner's syndrome. All other system examinations were normal.

Her MRI of cervical spine (fig 01) showed a flow void in the spinal canal extending from the $\mathrm{C} 2$ to $\mathrm{C} 6$ level and from the beginning of the right VA form SCA upto the foramen transverserium of C6. Her CT angiogram of the neck vessels (fig 02) showed that her right VA was dilated, tortuous, irregular shaped. It had entered the spinal canal with a narrow part, and then it had again dialated and compressed the dural tube upto the C2 level. It was draining into the right IJV. The distal part of VA was atritic but had communication with the cerebral vessels. An duplex study of the neck vessels was done. There was abnormal blood flow over the malformed artery in the root of the neck.

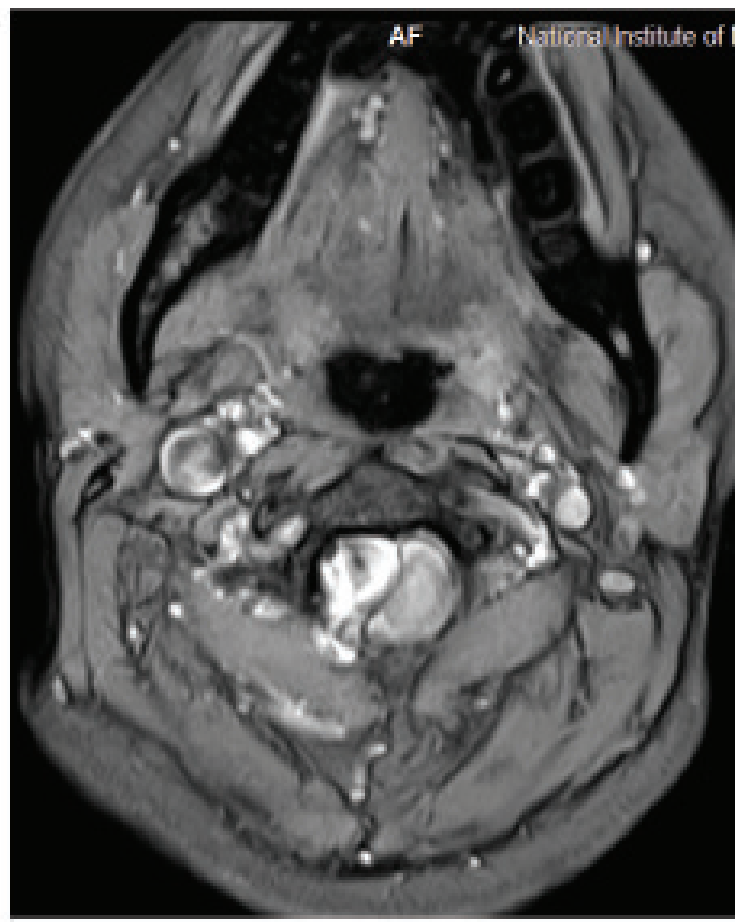

Fig.-1: A. Pre op MRI T1 Sagittal, B. Preop MRI Axial Contrast 

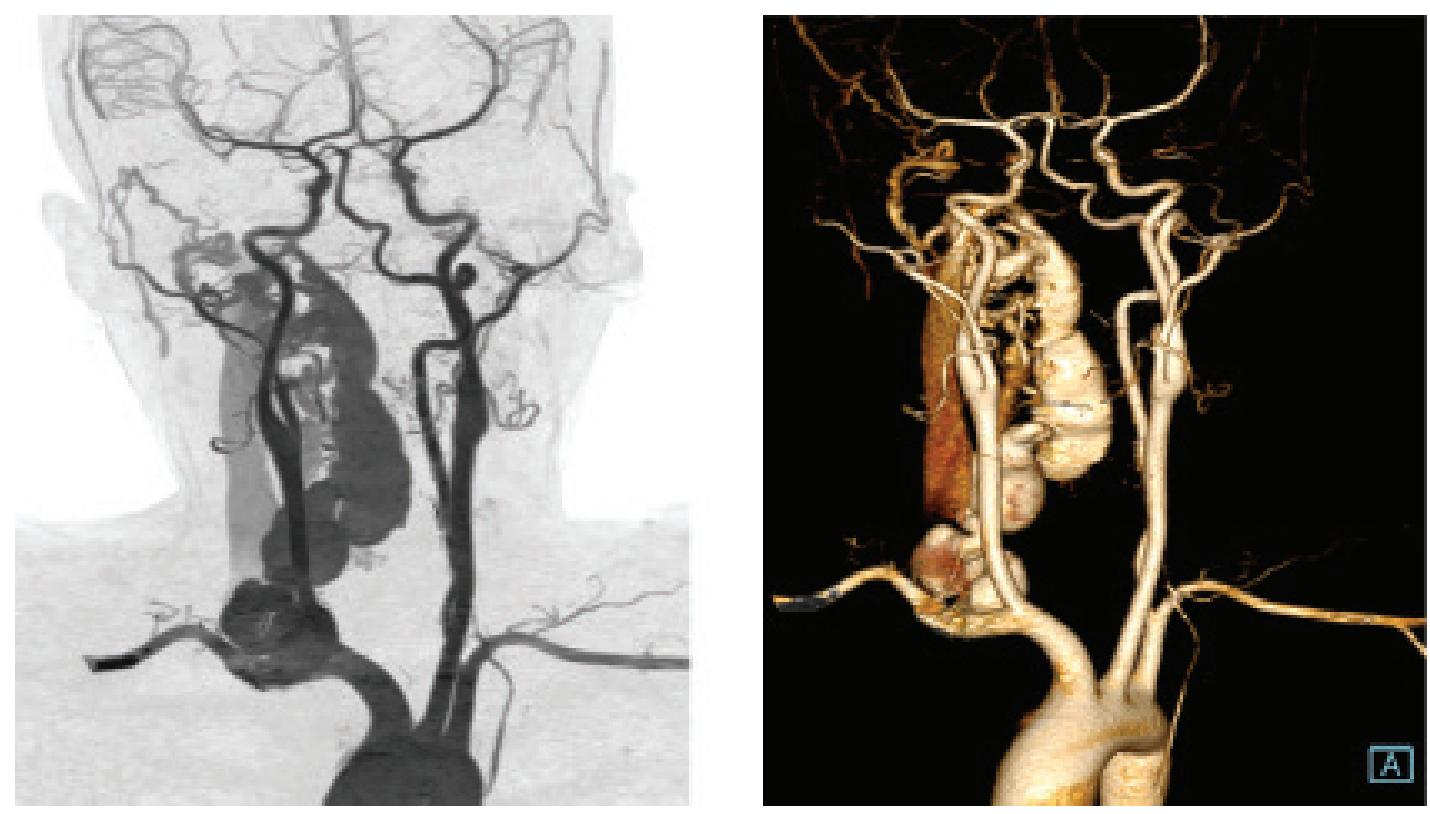

Fig.-2: Pre op CTA. Showing large AVF in the Spinal canal. The rt. IJV has high flow evidenced by large Diameter

The patient had undergone surgery. An oblique and linear incision was given at the neck along the sternocleidomastoid muscle. The right SCA was exposed and was traced laterally. The AVM was seen arising from the second part of the SCA. The neck was narrow and then it had dilated and ascended to enter through the right. Intervertebral foramen at C6/7 level with a narrow waist (Fig 03). The AVM was ligated at the origin and near the entry point at the Intervertebral foramen. It then collapsed and blood flow was absent, evidenced by no flow in intra-opeartive Doppler study.

At the $5^{\text {th }}$ POD the patient said that she started to have more power in the left side. After two weeks,

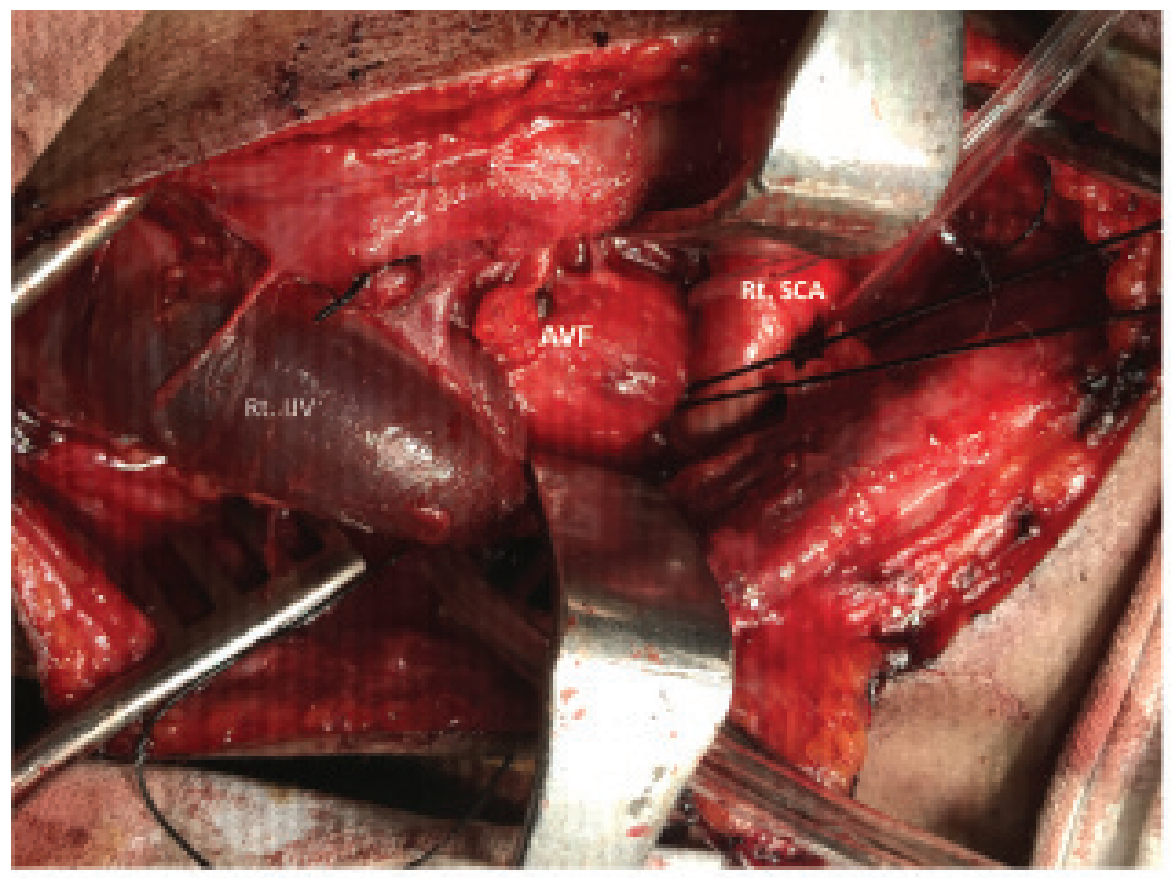

Fig.-3 : Intraoperative view of ligated AVF 
she gained enough strength to sit with support. At the end of one month she could walk with help from her mother.

After $1 \frac{1}{2}$ months, her CT angiogram and MRI was repeated. The MRI of Cervical spine (fig 05) showed collapse of the superior portion of the AVF, But there was dilated part in the lower part of the cervical part of the AVM . The CTA (fig 04) also showed narrowing of the AVF with retrograde filling of the right VA. The Rt. IJV had become narrow as well with reduced flow. The patient was scheduled for a second surgery for exploration of the part of AVF in the spinal canal. But the patient wanted to wait as she had much improvement. They were then discharged from the hospital and was scheduled for follow-up after six months. They came after six months and she was able to walk without support.
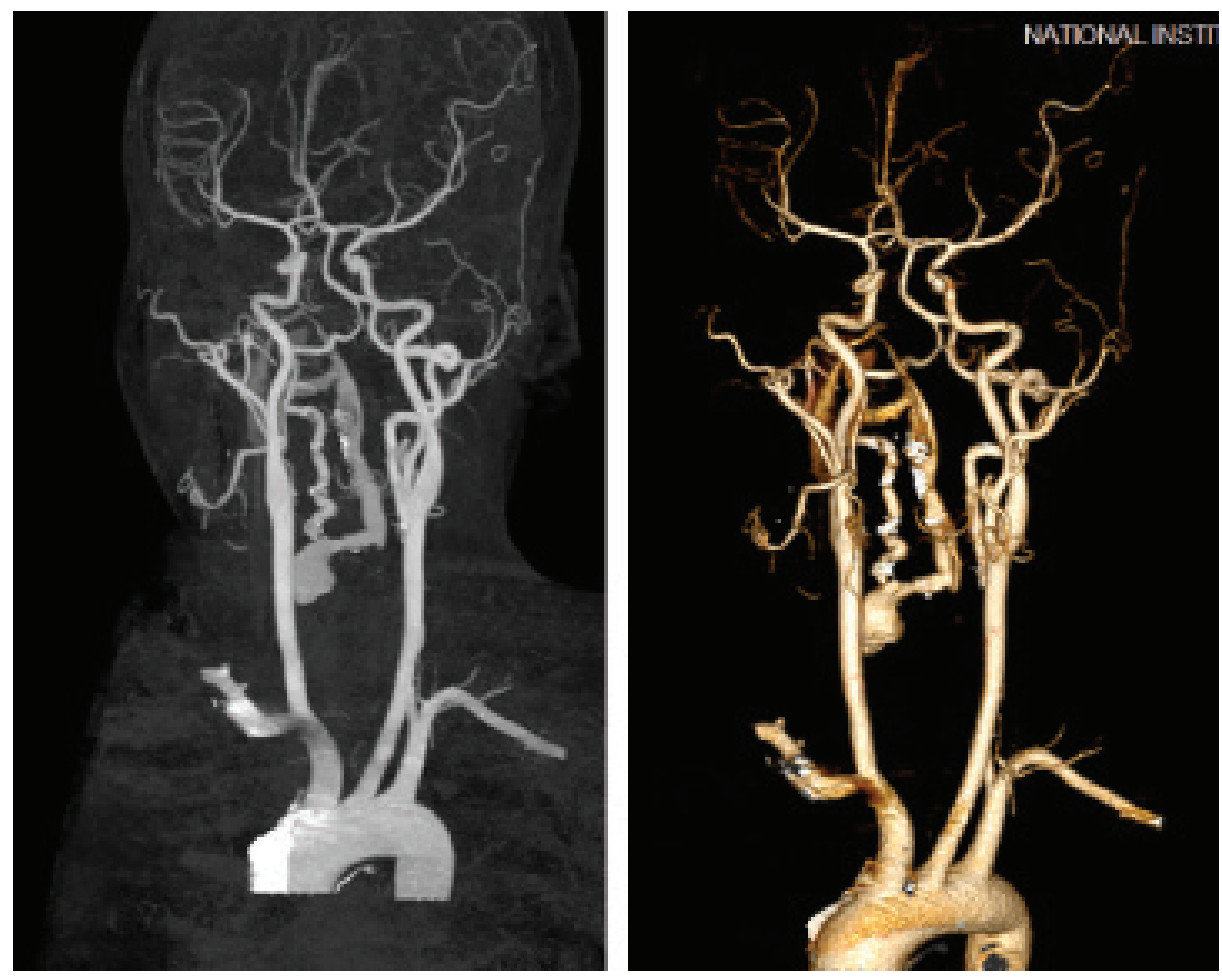

Fig.-4: Post opearative CTA : Showing Narrowing of the interFistula and decrease flow iof the e Rt. IJV. The Rt. $V A$ is seen filled with retrograde flow.
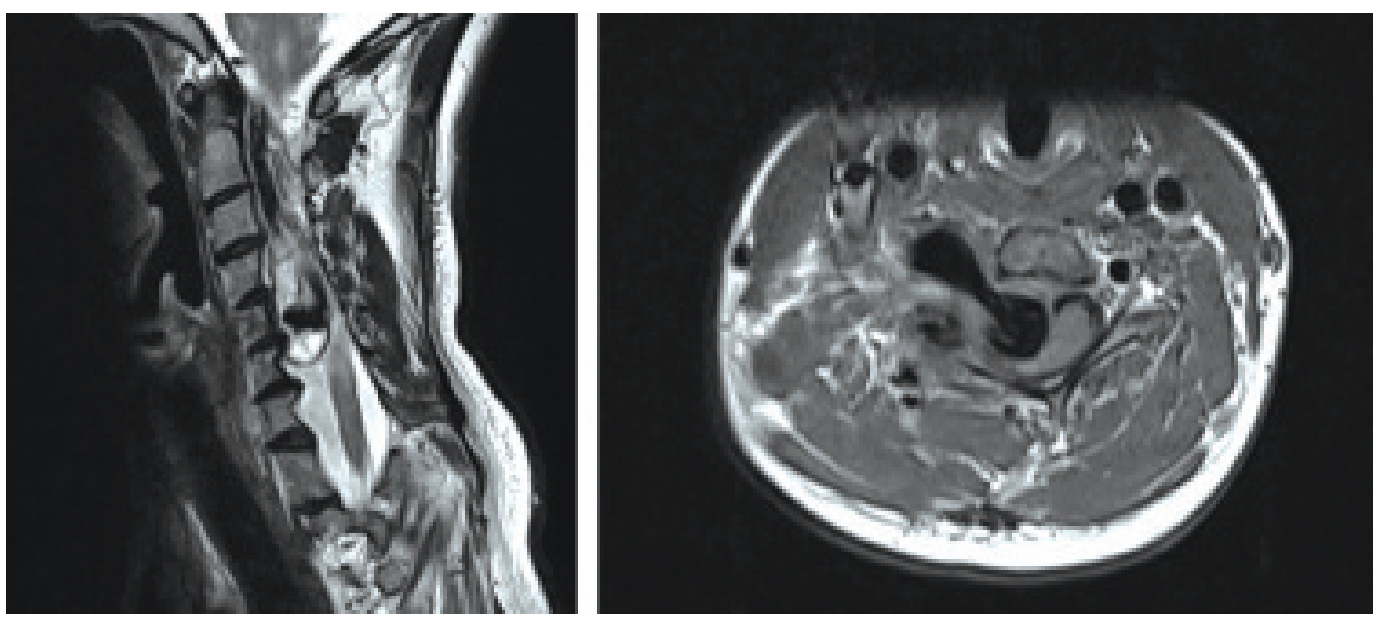

Fig.-5: Post op MRi showing narrowing of the Intra spinal AVF (6wees later) 


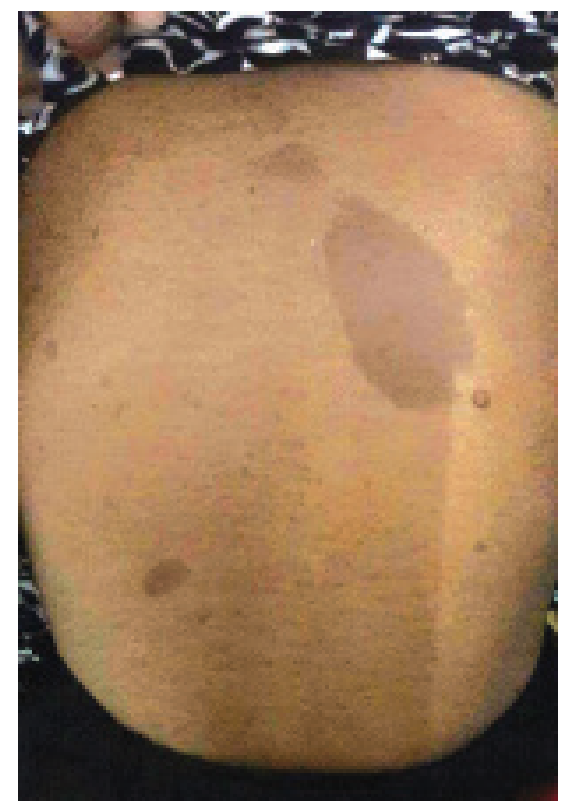

Fig.-6: Café au lait spots at the back

\section{Discussion:}

According to Kring et al. when SVM is suspected, MRI should constitute the first diagnostic modality. Using a small set of relevant sequences, the radiologist is able to detect intramedullary pathologies such as intramedullary hemorrhages, cavernomas, edema or venous congestion, extramedullary intradural alterations (such as dilated vessels or subarachnoidal hemorrhage) or potential extradural manifestations of SVM (such as associated hemangiomas). Moreover, most clinical differential diagnoses (tumor, degenerative diseases of the spine and cord compression) can be ruled out ${ }^{2}$. In our patient, the first investigation was also MRI of cervical spine.

Amirjamshidi had reported about a Vertebral artery AVF which had thrill in the neck ${ }^{3}$. Our patient had also had thrill in the neck. They had treated the AVF by trapping and embolization. In our case we had also trapped the AVF in the neck.

Defreyne et al had reported a similar case of vertebral artery AVM at C6 level, which had ruptured and caused tetraplegia with a complete sensory deficit below the level of C2 on the right and C4 on the left side ${ }^{7}$. Our patient also had similar picture with tetraparesis. But in our patient the AVM did not rupture. Geering has suggested that these malformations are rare except traumatic origin and may result in cardiac failure ${ }^{5}$. In our patient there was no history of trauma or any signs or symptoms of cardiac failure.
Lawson in his series had reported of a patient with paresthesia followed by quadriplegia, apparently from spinal cord ischemia. He also mentioned of local compression which had obliterated the bruit $^{8}$. In our patient the patient had quadriplegia which had improved one and half month following surgery also the bruit. Dieng reported a giant AVM at the neck ${ }^{9}$ but our patient's AVM was small compared to that.

Clinically, the AVMs caused only slight swelling of the soft tissues and were associated with a cutaneous discoloration similar to a port-wine stain. Warmth and pulsatility indicated rapid vascular circulation in the $\mathrm{AVMs}^{10}$. These may be accompanied with neurofibromatosis ${ }^{1}$ or other neurocutaneous syndromes ${ }^{11}$. Cluzel had reported two such cases $^{12}$.Our patient also had dark spots in the back and abdomen but no tumours in the brain. Our patient had erosion of the pedicle on the right side from $\mathrm{C} 2$ to C6 with dilatation of the intervertebral foramen. Brooks also had reported three such cases ${ }^{11}$.

Most traumatic vertebral fistulas involve the long second portion (intraforaminal), whereas spontaneous fistulas usually involve the third portion (where the artery leaves the foramen of the atlas to where it enters the foramen magnum $)^{13}$. But in our patient, the fistula had started from the origin of the Rt. VA and had extended to the level of $\mathrm{C} 1$.

Most AVF of the VA is difficult to approach by direct surgical approach due the deep placement of the vertebral artery. So they can easily be treated with endovascular embolization ${ }^{13}$. Yoshida had also reported of endovascular procedure ${ }^{1}$. Our patient was also referred for endovascular procedure, but the patient was unable to bear the high cost. However, the surgical procedure went smoothly and the result was quite satisfactory. The scar was also not that visible.

\section{Conclusion:}

Rt. Vertebral artery AVF inside the spinal canal is an unusual cause of quadriparesis. Its diagnosis is difficult and can be confirmed with CT Angiogram. it can be ligated safely at the neck and this results in collapse of the AVF. The patient had symptomatic improvement.

\section{References:}

1. Yoshida S, Nakazawa K, Oda Y. Spontaneous Vertebral Arteriovenous Fistula -Case Report. Neurologia medicochirurgica. 2000;40(4):211-5. 
2. Krings T, Mull M, Gilsbach JM, Thron A. Spinal vascular malformations. European Radiology. 2005;15(2):267-78.

3. Amirjamshidi A, Abbassioun K, Rahmat H. Traumatic aneurysms and arteriovenous fistulas of the extracranial vessels in war injuries. Surgical Neurology. 2000;53(2):136-45.

4. Ross DA, Olsen WL, Halbach V, Rosegay H, Pitts LH. Cervical root compression by a traumatic pseudoaneurysm of the vertebral artery: case report. Neurosurgery. 1988;22(2):414-7.

5. Geering J, Rutishauser M, Wyler F, Fliegel C. Arteriovenous malformation of the vertebral artery. Pediatric Radiology. 1976;4(4):254-6.

6. Greenberg J. Spontaneous arteriovenous malformations in the cervical area. Journal of neurology, neurosurgery, and psychiatry. 1970;33(3):303-9.

7. Defreyne L, Achten E, Vandekerckhove T, Kunnen M. Rupture of a cervical spinal cord arteriovenous malformation: a rare complication of endovascular embolization. European Radiology. 1999;9(4):734-7.
8. Lawson TL, Newton TH. Congenital Cervical Arteriovenous Malformations. Radiology. 1970;97(3):565-70.

9. Dieng PA, Ba PS, Gaye M, Diatta S, Diop MS, Sene E, et al. Giant Arteriovenous Malformation of the Neck. Case Reports in Vascular Medicine. 2015;2015:3.

10. Gelbert F, Riche MC, Reizine D, Guichard JP, Assouline E, Hodes JE, et al. MR imaging of head and neck vascular malformations. Journal of Magnetic Resonance Imaging. 1991;1(5):579-84.

11. Brooks BS, El Gammal T, Beveridge W. Erosion of vertebral pedicles by unusual vascular causes. Neuroradiology. 1982;23(2):107-12.

12. Cluzel P, Pierot L, Leung A, Gaston A, Kieffer E, Chiras J. Vertebral arteriovenous fistulae in neurofibromatosis: report of two cases and review of the literature. Neuroradiology. 1994;36(4):321-5.

13. Halbach VV, Higashida RT, Hieshima GB. Treatment of vertebral arteriovenous fistulas. American journal of neuroradiology. 1987;8(6):1121-8. 